\title{
Estratégia\&Negócios
}

ISSN 1984-3372

http://www.portaldeperiodicos.unisul.br/index.php/EeN/

\section{MENSURAÇÃO DA SATISFAÇÃO DE CLIENTES COM SERVIÇOS DE FAST FOOD}

\section{MEASUREMENT OF SATISFACTION CUSTOMERS WITH FAST FOOD SERVICES}

\section{Daiane Lindner Radons}

Mestranda em Administração pela Universidade de Santa Maria - UFSM.

E-mail: daialindner@yahoo.com.br

\section{Carolina Cunha Torres}

Acadêmica em Administração pela Universidade de Santa Maria - UFSM.

E-mail: caroltorrescp@gmail.com

\section{Paulo Sérgio Ceretta}

Professor adjunto da Universidade de Santa Maria - UFSM.

E-mail: ceretta@smail.ufsm.br

Recebido em 11/04/2012. Aprovado em 27/09/2012. Disponibilizado em 04/03/2013. Avaliado pelo Sistema double blind review

R. eletr. estrat. neg., Florianópolis, v.5, n.3, p. 122-150, set./dez. 2012 http://portaldeperiodicos.unisul.br/index.php/EeN/index
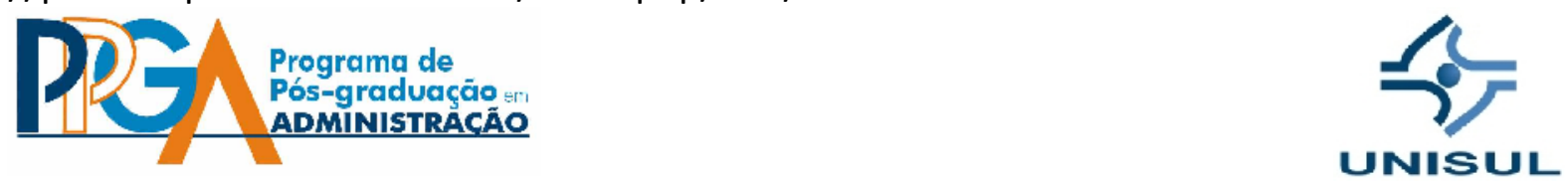

CCopyright 2008 UNISUL-PPGA/Estratégia e Negócios. Todos os direitos reservados. Permitida citação parcial, desde que identificada a fonte. Proibida a reprodução total. Em caso de dúvidas, consulte o editor: ademar.unisul@gmail.com; (48) 3229-1932. 


\section{RESUMO}

A satisfação dos clientes tornou-se um dos aspectos mais relevantes para as empresas devido ao aumento das exigências dos consumidores que passaram a usufruir de uma crescente oferta de produtos e serviços. Nesse sentido, vários estudos foram realizados visando a criar e aperfeiçoar modelos, como o Swedish Customer Satisfaction Index, American Customer Satisfaction Index e European Customer Satisfaction Index, para identificar o nível de satisfação dos clientes. O presente artigo tem como objetivo mensurar o nível de satisfação dos clientes com o serviço de fast food, considerando as variáveis referentes a expectativas dos clientes, qualidade percebida, imagem, valor percebido, reclamações e lealdade, que fazem parte do Índice Europeu de Satisfação do Consumidor. A pesquisa realizada é de caráter descritivo e a coleta de dados foi obtida por meio de questionário, composto por 30 questões, aplicado a 210 universitários na cidade de Santa Maria/RS. Para análise dos dados, foi utilizado o método PLS-PM. Os resultados encontrados evidenciam que a variável valor percebido foi a que mais influenciou na satisfação dos clientes, seguida das variáveis qualidade percebida, imagem e expectativa dos clientes. A maioria das hipóteses da pesquisa foram comprovadas, dessa forma, o modelo utilizado demonstrou ser adequado para verificar a satisfação dos clientes em relação aos serviços de fast food.

Palavras-chave: Fast-food. Partial Least Squares. Satisfação de clientes.

\section{INTRODUÇÃO}

Nas últimas décadas, a abertura do mercado nacional a empresas estrangeiras, assim como o crescimento da economia brasileira, contribuíram para o aumento da concorrência. Dessa forma, os consumidores têm se tornado cada vez mais exigentes em função do aumento de ofertas de produtos e serviços. Esse fato faz com que as empresas busquem formas de satisfazer os consumidores, atendendo as suas necessidades com qualidade. Alguns autores abordam que a lealdade é resultado da satisfação dos valores e expectativas do usuário alcançada a partir do processo de compra ou consumo de produtos (SHETH; MITTAL; NEWMAN, 2001; MOWEN; MINOR, 1998). Assim, o processo de recompra terá resultados positivos para a organização. 
Nesse contexto, tornou-se relevante identificar os fatores que determinam a satisfação do consumidor. Vários estudos realizados objetivaram descobrir índices de mensuração da satisfação dos clientes (DEVLIN; DONG; BROWN, 1993; WITTINK; BAYER, 1994). O primeiro índice, Swedish Customer Satisfaction Index (SCSI) foi desenvolvido em 1989, na Suécia. Posteriormente, estudos semelhantes foram realizados na Alemanha, Israel, Taiwan e Nova Zelândia. Em 1994, foi lançado o American Customer Satisfaction Index (ACSI), que serviu de base para o desenvolvimento, na Europa, do European Customer Satisfaction Index (ECSI). O Índice Europeu de Satisfação teve suas bases definidas pela European Foundation for Quality Management (EFQM) e a CSI University Network, sendo que doze países europeus aderiram ao modelo (LEITE; FILHO, 2007).

Partindo das premissas do Índice Europeu de Satisfação do Consumidor na Europa, o presente trabalho examinou empiricamente o modelo no Brasil, especificamente em uma universidade na região central do Estado do Rio Grande do Sul. Considerando que a avaliação da satisfação é fundamental para a empresa identificar seu desempenho, assim como alcançar a retenção de clientes e maiores lucros, esse trabalho objetivou identificar a satisfação dos consumidores com os serviços de fast food, que é um ramo alimentício em ascensão, devido às tendências da modernidade. As variáveis expectativas dos clientes, qualidade percebida, imagem, valor percebido, reclamações e lealdade foram levadas em consideração na mensuração da satisfação.

Estudos dessa natureza propiciam contribuições relacionadas com o nível de satisfação dos consumidores e quais dimensões são mais relevantes na decisão de compra de determinado produto ou serviço, assim, as organizações podem centrar esforços nas variáveis mais importantes para os consumidores, a fim de satisfazê-los e alcançar melhores resultados, visando a garantir sua sobrevivência no ambiente concorrencial.

O artigo está estruturado de forma a contemplar breve descrição do índice utilizado, conceituação de satisfação e de cada construto enfatizado no modelo, assim como caracterização dos serviços de fast food. Na sequência, os procedimentos metodológicos são considerados e por fim, são apresentados os resultados e as considerações finais do estudo. 


\section{SATISFAÇÃO DOS CLIENTES}

A satisfação dos clientes é um tema amplamente abordado em pesquisas e se mostra em evidência no meio acadêmico. Nessa perspectiva, o referencial teórico compreende conceituação de fast food, definições de satisfação de cliente, bem como as relações entre a mesma e outras dimensões teóricas como expectativa dos clientes, qualidade percebida, imagem, valor percebido, reclamações e lealdade. O modelo utilizado nesse estudo, Índice Europeu de Satisfação do Consumidor, também é especificado nessa seção.

Para Anderson e Fornell (1994), a satisfação é uma avaliação contínua a respeito da habilidade de uma marca em proporcionar os benefícios que o cliente busca ao longo do tempo. A satisfação do cliente é conceituada a partir de duas formas: satisfação específica em uma transação e satisfação acumulada (BOULDING et al., 1993). A concepção da satisfação específica a uma transação serve para a avaliação de situações específicas, enquanto a visão cumulativa é mais adequada quando o consumidor possui experiências passadas com determinado serviço (MILAN; TREZ, 2005).

A satisfação do cliente pode ser compreendida como uma comparação do que era esperado e as percepções de desempenho (CHITTY; SOUTAR, 2004). Oliver (1997) compreende a satisfação como a resposta de plenitude do consumidor, isto é, um julgamento que um produto ou serviço tem como característica, em função de ter proporcionado ou estar proporcionando um nível agradável de perfeição. A satisfação impacta no desempenho organizacional e segundo Fornell (1992, p. 18) os "clientes satisfeitos são um ativo para a organização. Mudanças na satisfação são consequências de decisões passadas e predizem o desempenho futuro."

A partir da concepção da satisfação como preditora do desempenho organizacional, Anderson e Mittal (2000) desenvolveram a Cadeia Satisfação-Lucro (Figura 1), a qual mostra que as empresas que buscam satisfazer seus clientes obtêm lucros significativos. Conforme a cadeia, a satisfação geral é função de vários atributos e a gestão das avaliações desses atributos pode conduzir ao aumento da satisfação, considerando que quanto maior a satisfação geral, maior a recompra e maior a retenção de clientes, o que resulta em maiores lucros para a empresa. No entanto, os autores da cadeia atentam que existem resultados contraditórios, em decorrência que a ligação de todos os pontos da cadeia são assimétricos e não lineares. 
Figura 1 - A Cadeia Satisfação - Lucro

Fonte: Anderson e Mittal (2000, p. 108).

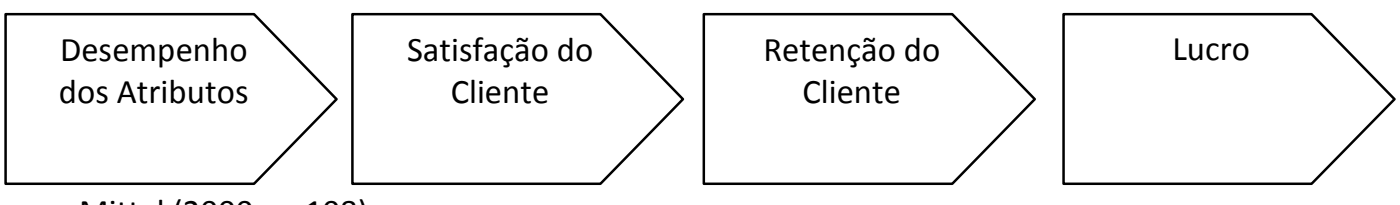

Assim, as empresas buscam alcançar a satisfação de clientes, pois ela conduz à retenção de clientes e à formação de lealdade (OLIVER, 1997) e permite maximizar o lucro da organização. Então, o alcance da lealdade é um dos desafios das organizações, as quais estão imersas no ambiente instável e de expressiva concorrência em que o consumidor tem a possibilidade de escolha em meio a uma diversidade de produtos e serviços, perpassando pela análise da satisfação.

\section{1 ÍNDICE EUROPEU DE SATISFAÇÃO DO CONSUMIDOR E DEFINIÇÃO DOS CONSTRUTOS}

Em decorrência das constantes mudanças no meio empresarial e do ambiente acirradamente competitivo, estudos realizados focaram o desenvolvimento de indicadores para mensurar a satisfação do cliente. Um deles é o European Customer Satisfaction Index (ECSI), traduzido como Índice Europeu de Satisfação do Consumidor, elaborado a partir do Swedish Customer Satisfaction Index (SCSB) ou Barômetro Sueco de Satisfação do Consumidor (FORNELL et al., 1996; LOUGHLIN; COENDERS, 2002).

O Barômetro Sueco de Satisfação do Consumidor foi criado em 1989 e consistiu no primeiro índice nacional de medição da satisfação do consumidor para análise de compras e consumo de produtos e serviços, sendo incluídas 130 companhias e 32 grandes indústrias suecas (FORNELL, 1992). O modelo em questão contempla dois fatores antecedentes à satisfação, a performance percebida (valor) pelo cliente com o produto ou serviço e a expectativa em relação à performance; e, dois fatores consequentes à satisfação, o comportamento e a fidelidade do consumidor. Para Rodrigues (2003), as expectativas afetam a satisfação em função de servirem como âncoras na evolução do processo, enquanto que a performance percebida engloba as recentes experiências do consumidor, considerando que a expectativa é gerada antes da experiência de consumo. 
Posteriormente ao índice sueco, o American Customer Satisfaction Index (ACSI) ou Índice Americano de Satisfação do Consumidor foi desenvolvido, em 1994, pela University of Michigan Business School and ASQ, tendo como base o modelo sueco, incluindo 200 companhias e 35 indústrias americanas (RODRIGUES, 2003). Considerando os seis construtos desse modelo (expectativa do consumidor, qualidade percebida, valor percebido, satisfação, índice de reclamações e fidelidade), Johnson et al. (2001) destaca que as principais diferenças entre os dois modelos são as adições da qualidade percebida como construto diferente de valor percebido e implementação das medidas das expectativas do consumidor.

O Índice Europeu de Satisfação do Consumidor, por sua vez, foi desenvolvido em 2000, sendo que estudos planejados foram realizados em 10 países europeus. Esse índice representa uma variação do ACSI, embora os construtos expectativa do consumidor, qualidade percebida, valor percebido, satisfação e fidelidade do consumidor são abordados da mesma forma pelos dois modelos.

O modelo europeu difere do americano pelo fato que enfatiza a fidelidade do cliente, incluindo a probabilidade de retenção, recomendação da empresa ou marca, e a probabilidade de aumentar a quantia de compras dos consumidores (JOHNSON et al., 2001). O Índice Europeu de Satisfação do Consumidor compreende um modelo de equações estruturais formado por uma série de construtos latentes que relacionam a satisfação dos clientes a suas causas e consequências (KRISTENSEN; MARTENSES; GRONHOLDT, 2000). Na Figura 2, é apresentado o modelo utilizado.

Figura 2 - Modelo estrutural de satisfação dos clientes

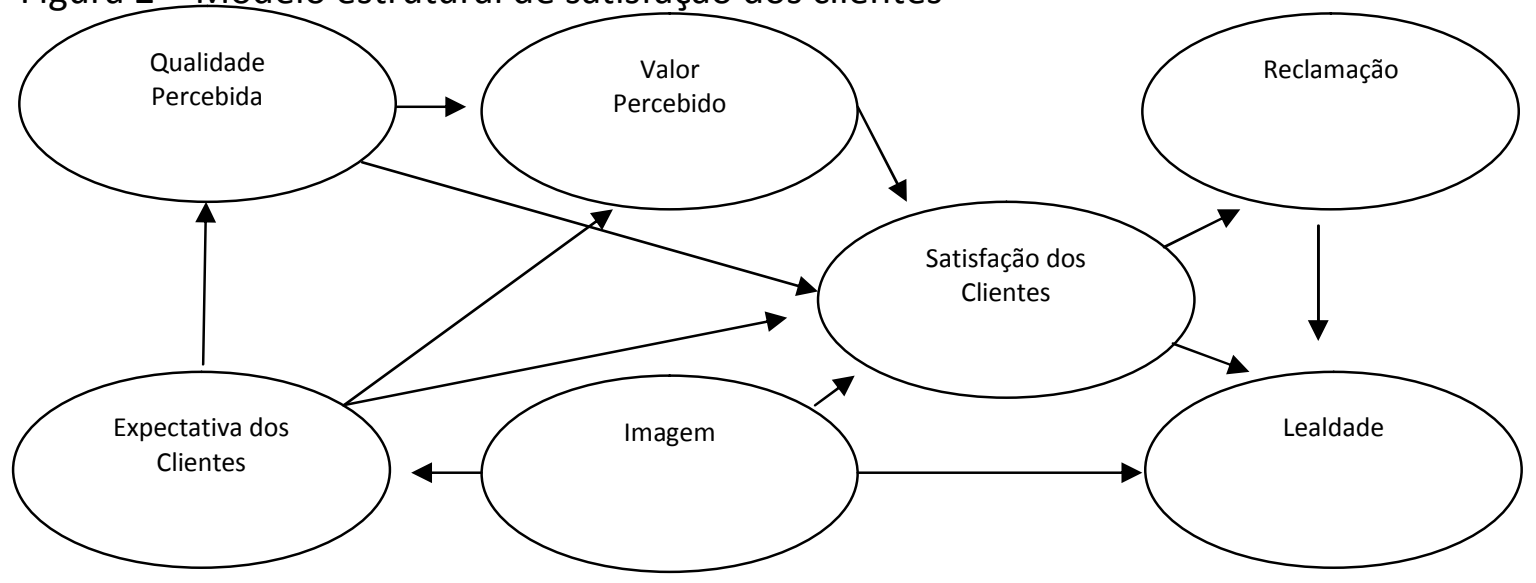

Fonte: Tenenhaus et al. (2005, p. 161). 
Conforme verificado na Figura 1, a satisfação de clientes está envolvida num processo de causa e consequências. Os construtos antecedentes (expectativa dos clientes, qualidade percebida, imagem e valor percebido), conhecidos como causa, determinam a satisfação do cliente, enquanto que os construtos consequentes (reclamações e lealdade) surgem a partir da satisfação dos clientes (FORNELL, 1992). Considerando as premissas e conclusões do Índice Europeu de Satisfação do Consumidor na Europa, o presente trabalho examinou empiricamente o modelo no Brasil, utilizando as hipóteses sustentadas pelo modelo, que são apresentadas e discutidas na sequência, assim como os construtos dos quais elas fazem parte. Tais hipóteses já foram testadas anteriormente em outros estudos (LEITE; FILHO 2007; LOPES; PEREIRA; VIEIRA, 2009) e na presente pesquisa se pretendeu testar empiricamente o modelo no contexto de uma cidade da região central do Rio Grande do Sul, a fim de examinar o nível de satisfação dos clientes, considerando os serviços de fast food.

\subsubsection{Imagem}

A imagem da empresa é compreendida como a impressão criada na mente dos consumidores (NGUYEN; LEBLANC, 2001). Sendo relevante para as empresas, a imagem pode ser definida, segundo Barich e Kotler (1991, p. 95), como "a soma das crenças, atitudes e impressões que uma pessoa ou grupo têm de um objeto, uma pessoa, um lugar, uma marca, um produto ou uma empresa". Webster Jr. (1994) considera a imagem positiva da marca ou nome da instituição uma das mais poderosas formas de diferenciação de um produto/serviço, sendo impossível para um concorrente duplicá-la.

Conforme Deschamps e Nayak (1996, p. 83) a imagem é relevante para a percepção da empresa por parte das pessoas, pois "o consumidor inicia seu processo de compra, implícita ou explicitamente, com uma avaliação da imagem". Fortalecendo essa ideia, Andreassen e Lindestad (1998) afirmam que a imagem é a âncora psicológica que afeta as expectativas, bem como a satisfação e a fidelidade do consumidor. Nesse sentido, a Hipótese 1 se refere à imagem e satisfação dos consumidores: a imagem influencia positivamente a satisfação dos clientes. 


\subsubsection{Expectativa dos clientes}

A expectativa do cliente é considerada como a experiência anterior dele com os produtos e serviços de uma empresa, bem como a percepção da propaganda (JOHNSON et al., 2001). Estudos realizados com modelos convencionais de satisfação do cliente têm enfatizado uma estrutura em que os clientes apresentam expectativas específicas sobre suas interações com a empresa (SCHNEIDER, 1999). Esse autor ainda defende que ao encontrar tais expectativas, a empresa pode satisfazer o cliente.

No modelo considerado, as expectativas são determinantes da satisfação do consumidor, uma vez que as expectativas exprimem tanto a experiência prévia de consumo do cliente com a oferta do fornecedor (considerando as informações não experienciais, de fontes como propaganda e comunicação boca a boca), como uma previsão da habilidade do fornecedor em entregar qualidade no futuro (URDAN; RODRIGUES, 1999). Então, levando em conta o seu caráter predizente de qualidade futura, supõe-se que as expectativas tenham efeito positivo sobre a satisfação global. Ademais, acredita-se que as expectativas sejam positivamente relacionadas à qualidade percebida e, em decorrência, ao valor percebido, porque o conhecimento do cliente deve ser tal, que suas expectativas ilustrem a qualidade corrente. A partir dessas considerações, a Hipótese 2 é apresentada: a expectativa dos clientes influencia positivamente a satisfação dos clientes.

\subsubsection{Qualidade percebida}

Há pouca convergência a respeito da definição de qualidade (HUFF; FORNELL; ANDERSON, 1994). Dessa forma, Grönroos (1990) e Fornell (1991) abordam que o mais importante é a definição do que seja qualidade sob a perspectiva do cliente. Dessa forma, qualidade é conformação às especificações do cliente, e o que o cliente percebe como qualidade é o mais relevante para as organizações.

A satisfação do consumidor pode ser utilizada para avaliar e intensificar o desempenho de empresas, indústrias e até economias nacionais, a partir da mensuração da qualidade dos bens e serviços (FORNELL et al, 1996). Por a qualidade de serviços ser de difícil mensuração, ela é 
avaliada de forma parcial, em função da natureza mais abstrata do serviço, resultante de sua intangibilidade e demais singularidades (VEIGA, 2000). Em contraste, a qualidade de bens pode ser medida de forma objetiva, por meio de indicadores como durabilidade e número de defeitos.

Dessa forma, a qualidade pode ser vista como o potencial intrínseco aos atributos do produto ou serviço a fim de prover satisfação, ressaltando que a qualidade percebida é dependente da função de utilidade para o cliente em relação ao nível de desempenho ou qualidade fornecido (ANDERSON; FORNELL, 1994). Corroborando a importância da qualidade na avaliação da satisfação, Moreira (1996) afirma que a mensuração da qualidade está diretamente relacionada ao grau de satisfação dos clientes. Partindo desse pressuposto, a Hipótese 3 formulada é: a qualidade percebida influencia positivamente a satisfação dos clientes.

\subsubsection{Valor percebido}

Para Zeithaml (1988, p. 14), valor percebido "é a avaliação geral pelo consumidor da utilidade de um produto baseado em percepções do que é recebido e do que é dado". Nessa perspectiva, o valor percebido é o nível entendido de qualidade do produto em relação ao preço pago, incorporando ao valor percebido o fator preço, que reforça a comparabilidade de resultados entre fornecedores, ramos de atividades e setores.

Gale (1996) faz uma comparação entre qualidade e valor percebido. Segundo o autor, qualidade percebida pelo mercado é definida como a opinião dos clientes sobre os produtos ou serviços, comparados com os da concorrência. Já o valor percebido é a qualidade percebida pelo mercado relativo ao produto ou serviço. O valor percebido é um elemento que antecede a satisfação, que em conjunto com os construtos qualidade percebida e expectativas, possibilita mensurar a satisfação do cliente (NETO; COSTA; PESSOA, 2009). A partir dessa associação entre valor percebido e satisfação, a Hipótese 4 é sustentada: o valor percebido influencia positivamente a satisfação dos clientes. 


\subsubsection{Reclamações}

A maneira pela qual a empresa trata as reclamações dos seus clientes pode influenciar significativamente na satisfação com o serviço, bem como na lealdade. Dessa forma, ter uma estrutura e processos adequados para o atendimento de reclamações pode ser importante para que uma empresa tenha clientes mais satisfeitos e leais (LOPES; PEREIRA; VIEIRA, 2009).

Richins (1983) relata que o comportamento de reclamação tem sido analisado em termos de classe social, características de personalidade, gravidade do problema ou da insatisfação, ampliação da insatisfação para outras pessoas que não o reclamante, e a responsividade da empresa para aceitar a reclamação.

Segundo a teoria de voz-saída de Hirschman (1970), as consequências imediatas de maior satisfação do cliente são o decréscimo de suas reclamações e o incremento de sua lealdade. Quando insatisfeito, o cliente tem as opções de deixar o fornecedor e migrar para o concorrente, e de verbalizar as reclamações na tentativa de uma solução. Por isso, no modelo, supõe-se que o aumento da satisfação global deve reduzir a incidência de reclamações, o que permitiu a formulação da Hipótese 5: a satisfação dos clientes influencia positivamente as reclamações.

\subsubsection{Lealdade}

Conforme Oliver (1999), lealdade é definida como um comprometimento profundo em recomprar ou favorecer um produto ou serviço no futuro, causando repetição de marca ou compra de uma mesma empresa, embora influências situacionais e esforços de marketing tenham potencial para causar um comportamento de troca. Ainda, Jones e Sasser (1995) salientam que a lealdade do cliente pode ser compreendida como o sentimento de ligação ou de afeto para com os colaboradores de uma empresa e seus produtos.

Reichheld (2003) afirma que a lealdade dos clientes é que conduz à lucratividade para a empresa. Segundo o autor, a melhor forma de avaliar o quanto o cliente é leal diante de certa empresa, é medir o quanto essa empresa é recomendada pelos seus clientes. Dessa forma, clientes leais têm a possibilidade de trazer novos clientes para a empresa. 
Conforme mencionado na relação entre reclamações e satisfação, as consequências imediatas da promoção de maior satisfação do cliente conduzem ao decréscimo de suas reclamações e o incremento de sua lealdade. Assim, o modelo pressupõe que o aumento da satisfação global deve aumentar a lealdade do cliente. A partir do exposto, a Hipótese 6 é a seguinte: a satisfação dos clientes influencia positivamente a lealdade deles.

\subsubsection{Reclamações versus lealdade}

A última hipótese leva em consideração o relacionamento entre reclamações do cliente e sua lealdade. Fornell (1992) aponta que a direção e o sinal desse relacionamento dependem dos sistemas de serviço ao cliente e da solução de reclamações dada pelo fornecedor. Quando o relacionamento é positivo, significa que o fornecedor foi bem-sucedido em transformar um cliente que reclama em um cliente leal. Quando o relacionamento é negativo, o fornecedor atuou de tal forma que a situação negativa tornou-se ainda pior, contribuindo para a perda da lealdade do cliente. Assim, a Hipótese 7 consiste em: as reclamações influenciam positivamente a lealdade dos clientes.

\section{PROCEDIMENTOS METODOLÓGICOS}

A fim de atingir o objetivo proposto, foi realizada uma pesquisa de caráter descritiva, que segundo Hair et al. (2005, p. 86), "os planos de pesquisa descritiva em geral são estruturados e especificamente criados para medir as características descritas em uma questão de pesquisa."

Nesse estudo, utilizou-se o Índice Europeu de Satisfação do Consumidor, abordagem realizada por Tenenhaus et al. (2005), consistindo em um questionário como instrumento de coleta de dados. Para tanto, as afirmações foram traduzidas para o português e retraduzidas para o idioma inglês e, em seguida, um grupo de especialistas confirmaram a validade da escala. As questões foram adaptadas no sentido de utilizar o termo "fast food".

$\mathrm{O}$ instrumento foi composto de 30 questões referentes ao modelo europeu de satisfação, considerando as variáveis: expectativa dos clientes, qualidade percebida de produtos e serviços, imagem, valor percebido, lealdade e reclamações. A escala utilizada para as respostas 
varia de 1 (nota mínima) a 10 (nota máxima), sendo que o questionário ainda contou com questões nominais e ordinais para a identificação das variáveis que são normalmente usadas para segmentar. Nesta pesquisa, optou-se por considerar as variáveis demográficas e socioeconômicas, a fim de identificar o perfil dos respondentes.

O estudo buscou compreender a percepção dos alunos de graduação de diferentes cursos da Universidade Federal de Santa Maria - UFSM, na cidade de Santa Maria/RS, sobre a percepção da satisfação com o serviço de fast food. Foi selecionada uma amostra não probabilística de 210 acadêmicos regularmente matriculados na instituição.

A coleta de dados foi realizada nos meses de março, abril, maio e junho de 2011. Pela baixa ocorrência de dados faltantes no questionário, esses dados foram substituídos pela média. Para a obtenção dos resultados, foi utilizado o método multivariado Partial Least Squares Path Modeling (PLS-PM), desenvolvido por Wold (1974, 1982, 1985) para analisar dados de alta dimensão em um ambiente de baixa estrutura.

O PLS-PM é indicado para um estágio inicial do desenvolvimento teórico, testando e validando modelos exploratórios, além de adequar-se para pesquisas direcionadas à previsão. 0 modelo PSL-PM é definido por dois grupos de equações lineares, o modelo interno e externo. 0 modelo interno compreende as relações entre as variáveis latentes ou não observáveis. Já o modelo externo especifica as relações entre uma variável latente e suas variáveis manifestas ou observáveis.

A fim de avaliar o modelo reflexivo, utilizou-se o Rho D.G. e de acordo com Tenenhaus et al. (2005), valores acima de 0,7 são considerados satisfatórios. A avaliação da validade do modelo utilizado enfatiza dois subtipos: validade convergente e validade discriminante. A variância média extraída (AVE) é sugerida por Fornell e Larcker (1981) como critério de validade convergente. A validade discriminante pode ser mensurada por duas formas, o cross-loadings, que postula que o loading de cada indicador deve ser maior que todos os seus cross-loadings, e o critério de Fornell-Larcker (FORNELL; LARCKER, 1981), que compreende que o AVE de cada variável latente deve ser maior que a mais alta correlação quadrada da variável latente com qualquer outra variável. A validação do modelo interno é realizada pelo coeficiente de determinação $\left(R^{2}\right)$ das variáveis latentes. 


\section{ANÁLISE DOS RESULTADOS}

A amostra da pesquisa foi composta por 210 universitários, sendo 146 pessoas do sexo feminino (69,5 \%) e 64 do sexo masculino (30,5\%). Em relação à idade, 51,9\% dos respondentes encontram-se na faixa de até 20 anos, seguida de $45,7 \%$ dos acadêmicos que possuem de 21 a 30 anos, e, ainda 2,4\% dos estudantes têm mais de 31 anos. De acordo com a percepção dos questionados, a maioria $(52,4 \%)$ encontra-se na faixa de renda média, seguida da renda média alta $(19,2 \%)$ e da renda média baixa (18,8\%). A questão referente à renda não apresentou alternativas em forma de valor monetário, mas sim as opções renda alta, média e baixa. Como esperado, a maioria $(97,1 \%)$ dos respondentes se enquadra em nível superior incompleto ou completo.

Na questão referente ao local de preferência em que adquirem fast food, 155 pessoas preferem adquirir em lancherias (estabelecimento fixo), que equivale a $77,8 \%$ do total. Em segundo lugar, a franquia é o local predileto, com 15,7\% das respostas. Por último, 10,5\% das pessoas preferem adquirir de comerciantes ambulantes (local móvel, como trailers). Considerando a frequência com que os acadêmicos consomem fast food, verificou-se que $43,1 \%$ dos entrevistados consomem uma vez por semana; em segundo lugar, 36,8\% dos estudantes consomem uma vez por mês; e, 20,1\% consomem de 2 a 6 vezes por semana.

Através do teste de Kolmogorov-Smirnov e com correção de Lilliefors foi testada a normalidade das respostas de cada questão, considerando que o Kolmogorov-Smirnov é adequado para amostra superior a 50 (CORRAR et al., 2006). Os resultados obtidos pelo teste identificaram que todas as variáveis manifestas do estudo rejeitam a hipótese nula de normalidade (não seguem distribuição normal), considerando que o p-valor calculado é menor que o nível de significância alfa $(0,05)$. Esse fato fortalece a aplicação do método PLS-PM, que é uma técnica que não exige normalidade das variáveis.

A Tabela 1 apresenta o modelo estrutural utilizado na pesquisa com as respectivas médias para cada questão. As perguntas específicas do questionário (variáveis manifestas) estão relacionadas às respectivas variáveis latentes. A coluna observações compreende o número de observações obtidas para cada questão, totalizando 210 observações em todos os casos. Os dados faltantes foram substituídos pela média. Os valores de mínimo e máximo correspondem aos valores extremos que foram atribuídos às questões do instrumento de coleta de dados. A média se 
refere às médias obtidas com as respostas, enquanto que o desvio-padrão representa a variabilidade das respostas obtidas, considerando a média. Algumas variáveis foram retiradas por apresentarem baixo valor explicativo (v9, v21, v29).

Conforme os dados apresentados na Tabela 1, observa-se as maiores e menores médias obtidas no estudo, no intuito de verificar as questões que os consumidores pesquisados mais concordam ou discordam. A maior média $(7,80)$, considerando que a escala apresentava variação de 1 (nota mínima) a 10 (nota máxima), se refere à empresa ser estável e estabelecida (v2), tal afirmação faz parte do construto imagem. A segunda maior média foi obtida pela questão que compreende a possibilidade do consumidor indicar a empresa de fast food preferida para um amigo (v30), que pertence à dimensão lealdade e que atingiu o valor de 7,71 . Posteriormente, a afirmação sobre a qualidade esperada no momento em que o indivíduo opta por determinado fast food (v7) alcançou a terceira maior média $(7,44)$, a qual faz parte do construto expectativa do cliente.

A menor média $(5,02)$ foi obtida pela questão, inclusa no construto imagem, que compreende o papel social da empresa, ao mencionar se o consumidor percebe que a empresa preferida de fast food contribui com a sociedade (v3). A partir desses achados, é possível inferir que os consumidores estão atentos, em maior grau, com a imagem (se é estabelecida ou não) da empresa que presta o serviço e a qualidade esperada. Toni et al. (2006) afirmam que as imagens, enquanto representações, são capazes de influenciar e direcionar o comportamento das pessoas, sendo que a sua compreensão pelo consumidor constitui um importante trunfo para um melhor direcionamento das decisões de lançamento, aperfeiçoamento e desenvolvimento de estratégias de posicionamento de serviços. Ademais, a recomendação de empresas de fast food mostra-se uma prática recorrente entre os jovens pesquisados.

Tabela 1 - Estatística descritiva

\begin{tabular}{l|l|c|c|c|c|c}
\hline \multirow{2}{*}{ Variável Latente } & Variável Manifesta & Observações & Mínimo & Máximo & Média & Desvio padrão \\
\hline \multirow{5}{*}{ Qualidade } & v11 & 210 & 1,000 & 10,000 & 6,859 & 2,020 \\
\cline { 2 - 7 } & v12 & 210 & 1,000 & 10,000 & 6,826 & 1,915 \\
\cline { 2 - 7 } & v13 & 210 & 1,000 & 10,000 & 6,539 & 2,152 \\
\cline { 2 - 7 } & v14 & 210 & 1,000 & 10,000 & 7,082 & 2,095 \\
\cline { 2 - 7 } & v15 & 210 & 1,000 & 10,000 & 7,126 & 1,994 \\
\cline { 2 - 7 } & v16 & 210 & 1,000 & 10,000 & 6,605 & 2,224 \\
\cline { 2 - 7 } & v17 & 1,000 & 10,000 & 6,063 & 2,498 \\
\hline
\end{tabular}


Tabela 1 - Estatística descritiva

(conclusão)

\begin{tabular}{|c|c|c|c|c|c|c|}
\hline \multirow{3}{*}{ Expectativa clientes } & v7 & 210 & 1,000 & 10,000 & 7,443 & 1,962 \\
\hline & v8 & 210 & 1,000 & 10,000 & 7,097 & 2,371 \\
\hline & v10 & 210 & 1,000 & 10,000 & 6,824 & 2,786 \\
\hline \multirow{2}{*}{ Valor percebido } & v18 & 210 & 1,000 & 10,000 & 5,876 & 2,630 \\
\hline & v20 & 210 & 1,000 & 10,000 & 7,219 & 1,862 \\
\hline \multirow{8}{*}{ Imagem } & v1 & 210 & 1,000 & 10,000 & 6,756 & 2,003 \\
\hline & v2 & 210 & 1,000 & 10,000 & 7,804 & 2,201 \\
\hline & v3 & 210 & 1,000 & 10,000 & 5,019 & 2,655 \\
\hline & v4 & 210 & 1,000 & 10,000 & 6,130 & 2,459 \\
\hline & v5 & 210 & 1,000 & 10,000 & 5,707 & 2,751 \\
\hline & v6 & 210 & 1,000 & 10,000 & 6,862 & 2,928 \\
\hline & v22 & 210 & 1,000 & 10,000 & 6,541 & 2,472 \\
\hline & v23 & 210 & 1,000 & 10,000 & 6,678 & 2,601 \\
\hline \multirow{4}{*}{ Satisfação } & v19 & 210 & 1,000 & 10,000 & 7,063 & 1,995 \\
\hline & v24 & 210 & 1,000 & 10,000 & 7,015 & 1,878 \\
\hline & $\mathrm{v} 25$ & 210 & 1,000 & 10,000 & 6,965 & 1,814 \\
\hline & v26 & 210 & 1,000 & 10,000 & 6,894 & 1,944 \\
\hline \multirow{2}{*}{ Lealdade } & v28 & 210 & 1,000 & 10,000 & 6,337 & 2,302 \\
\hline & v30 & 210 & 1,000 & 10,000 & 7,710 & 2,292 \\
\hline Reclamação & v27 & 210 & 1,000 & 10,000 & 5,282 & 2,685 \\
\hline
\end{tabular}

Fonte: Elaborado pelos autores, 2012.

A fim de avaliar a confiabilidade da escala, ou seja, a consistência interna entre os múltiplos indicadores de um construto, adotou-se o cálculo do coeficiente Alpha de Cronbach para cada um dos construtos. O Alpha de Cronbach da variável Satisfação foi de 0,851 , considerado como muito bom e os dados para cada um dos fatores foram: Imagem - Alpha Cronbach = 0,811; Expectativa dos clientes - Alpha Cronbach =0,613; Qualidade - Alpha Cronbach $=0,892 ;$ Valor percebido - Alpha Cronbach $=0,699 ;$ Lealdade - Alpha Cronbach $=0,679$ (Tabela 2). Estes índices são considerados adequados, pois, de acordo com Malhotra (2001), aceitam resultados acima de 0,60. Assim, conclui-se que o instrumento possui uma consistência interna muito boa.

Tabela 2 - Confiabilidade do bloco

\begin{tabular}{l|c|c|c}
\hline Variável Latente & $\mathrm{N}^{\circ}$ de itens & Alfa de Cronbach & Rho de D.G. \\
\hline Imagem & 8 & 0,811 & 0,861 \\
\hline Expectativa dos clientes & 3 & 0,613 & 0,798 \\
\hline Qualidade & 7 & 0,892 & 0,917 \\
\hline Valor percebido & 2 & 0,699 & 0,891 \\
\hline Satisfação & 4 & 0,851 & 0,900 \\
\hline Reclamação & 1 & - & - \\
\hline Lealdade & 2 & 0,675 & 0,860 \\
\hline
\end{tabular}

Fonte: Elaborado pelos autores, 2012. 
O Rho de D. G também atesta a validade do modelo interno e, nesse estudo, todos os valores de Rho de D. G. confirmam a consistência interna do modelo utilizado, considerando que para Tenenhaus et al. (2005), um bloco será unidimensional quando o Rho de D. G. for superior a 0,7. Observa-se que o construto reclamação não apresentou resultados devido a conter somente uma questão no instrumento de coleta de dados, impossibilitando a análise.

Após a verificação da consistência interna, foi constatada a validade convergente (Tabela 3) por meio da variância média extraída (AVE), conforme sugere Fornell e Larcker (1981). A maioria dos valores AVE são superiores a 0,5 (exceto imagem) indicando validade suficiente para a pesquisa, uma vez que, para Hair et al. (1998), é necessário que a AVE dos construtos seja superior a 50\% para evidenciar validade convergente. A AVE de, no mínimo, 0,5 indica que a variável latente é capaz de explicar, em média, mais que metade da variância de seus indicadores. A variável latente imagem não possui valor convergente, explicando quase metade $(43,1 \%)$ das variáveis que a formam. Porém, ela possui valor discriminatório, uma vez que explica mais que qualquer outra variável.

Tabela 3-Validez convergente e discriminante (Correlações quadradas < AVE)

\begin{tabular}{|c|c|c|c|c|c|c|c|c|}
\hline 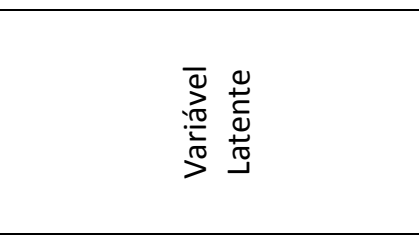 & 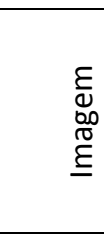 & 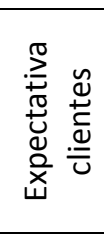 & $\begin{array}{l}\frac{0}{0} \\
\frac{\pi}{0} \\
\frac{.0}{\pi} \\
\frac{\pi}{2} \\
0\end{array}$ & 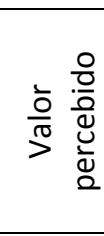 & 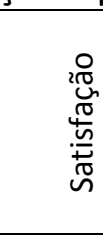 & 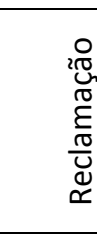 & $\begin{array}{l}\frac{0}{0} \\
\frac{\pi}{0} \\
\frac{0}{\pi} \\
\stackrel{\Xi}{\Xi}\end{array}$ & 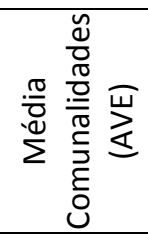 \\
\hline Imagem & 1 & 0,291 & 0,397 & 0,215 & 0,337 & 0,164 & 0,278 & 0,431 \\
\hline Expectativa clientes & 0,291 & 1 & 0,473 & 0,320 & 0,336 & 0,154 & 0,302 & 0,585 \\
\hline Qualidade & 0,397 & 0,473 & 1 & 0,560 & 0,551 & 0,311 & 0,339 & 0,611 \\
\hline Valor percebido & 0,215 & 0,320 & 0,560 & 1 & 0,567 & 0,316 & 0,349 & 0,767 \\
\hline Satisfação & 0,337 & 0,336 & 0,551 & 0,567 & 1 & 0,269 & 0,488 & 0,694 \\
\hline Reclamação & 0,164 & 0,154 & 0,311 & 0,316 & 0,269 & 1 & 0,324 & \\
\hline Lealdade & 0,278 & 0,302 & 0,339 & 0,349 & 0,488 & 0,324 & 1 & 0,754 \\
\hline Média Comunalidades (AVE) & 0,432 & 0,585 & 0,611 & 0,767 & 0,694 & & 0,754 & \\
\hline
\end{tabular}

Fonte: Elaborado pelos autores, 2012.

$\mathrm{Na}$ Tabela 4, são apresentadas as questões com suas respectivas cargas fatoriais (loadings) a fim de identificar o impacto de cada afirmação no construto a que pertence. Analisando a tabela, verifica-se que as cargas fatoriais de todas as variáveis manifestas são significativas, ao passo que apresentam razão crítica superior ao valor de 1,96. 
Tabela 4 - Cargas Fatoriais (loadings) Normalizadas e Estimadas pelo Bootstrap das Variáveis Manifestas

\begin{tabular}{|c|c|c|c|c|c|c|}
\hline Variável Latente & $\begin{array}{c}\text { Variáveis } \\
\text { manifestas }\end{array}$ & Loading & $\begin{array}{c}\text { Loading } \\
\text { (normalizado) }\end{array}$ & $\begin{array}{c}\text { Loading } \\
\text { (Bootstrap) }\end{array}$ & Erro padrão & $\begin{array}{c}\text { Razão crítica } \\
\text { (CR) }\end{array}$ \\
\hline \multirow{7}{*}{ Qualidade } & v11 & 0,082 & 0,140 & 0,082 & 0,006 & 13,545 \\
\hline & v12 & 0,074 & 0,126 & 0,073 & 0,006 & 12,517 \\
\hline & v13 & 0,087 & 0,148 & 0,086 & 0,007 & 13,099 \\
\hline & v14 & 0,087 & 0,147 & 0,087 & 0,006 & 15,486 \\
\hline & v15 & 0,060 & 0,102 & 0,060 & 0,008 & 7,114 \\
\hline & v16 & 0,093 & 0,159 & 0,093 & 0,007 & 13,836 \\
\hline & v17 & 0,105 & 0,179 & 0,106 & 0,009 & 11,794 \\
\hline \multirow{3}{*}{$\begin{array}{l}\text { Expectativa dos } \\
\text { clientes }\end{array}$} & v7 & 0,199 & 0,357 & 0,198 & 0,021 & 9,253 \\
\hline & v8 & 0,221 & 0,397 & 0,216 & 0,019 & 11,818 \\
\hline & v10 & 0,137 & 0,245 & 0,139 & 0,027 & 4,975 \\
\hline \multirow{2}{*}{ Valor percebido } & v18 & 0,283 & 0,591 & 0,280 & 0,014 & 20,225 \\
\hline & v20 & 0,196 & 0,409 & 0,198 & 0,015 & 12,883 \\
\hline \multirow{8}{*}{ Imagem } & v1 & 0,073 & 0,123 & 0,074 & 0,012 & 5,882 \\
\hline & $\mathrm{v} 2$ & 0,066 & 0,111 & 0,067 & 0,011 & 6,277 \\
\hline & v3 & 0,075 & 0,126 & 0,075 & 0,010 & 7,795 \\
\hline & v4 & 0,091 & 0,153 & 0,090 & 0,012 & 7,490 \\
\hline & v5 & 0,088 & 0,148 & 0,087 & 0,011 & 8,002 \\
\hline & v6 & 0,064 & 0,108 & 0,065 & 0,011 & 5,921 \\
\hline & $\mathrm{v} 22$ & 0,055 & 0,092 & 0,053 & 0,011 & 4,880 \\
\hline & v23 & 0,083 & 0,138 & 0,083 & 0,011 & 7,508 \\
\hline Variável Latente & $\begin{array}{c}\text { Variáveis } \\
\text { manifestas }\end{array}$ & Loading & $\begin{array}{c}\text { Loading } \\
\text { (normalizado) }\end{array}$ & $\begin{array}{c}\text { Loading } \\
\text { (Bootstrap) }\end{array}$ & Erro padrão & $\begin{array}{c}\text { Razão crítica } \\
\text { (CR) }\end{array}$ \\
\hline \multirow{4}{*}{ Satisfação } & v19 & 0,182 & 0,291 & 0,181 & 0,017 & 10,846 \\
\hline & v24 & 0,155 & 0,249 & 0,157 & 0,012 & 13,110 \\
\hline & v25 & 0,155 & 0,249 & 0,155 & 0,013 & 11,989 \\
\hline & v26 & 0,132 & 0,212 & 0,130 & 0,014 & 9,750 \\
\hline \multirow{2}{*}{ Lealdade } & v28 & 0,247 & 0,493 & 0,242 & 0,017 & 14,420 \\
\hline & v30 & 0,253 & 0,507 & 0,255 & 0,018 & 14,337 \\
\hline Reclamação & $\mathrm{v} 27$ & 0,372 & 1,000 & 0,374 & 0,010 & 35,389 \\
\hline
\end{tabular}

Fonte: Elaborado pelos autores, 2012.

Observa-se que o construto qualidade recebe maior impacto da variável clareza e transparência da informação fornecida pela empresa (v17). A expectativa dos clientes é mais influenciada pela questão que se refere ao consumidor esperar que a empresa ofereça produtos e serviços que atendam as suas necessidades (v8). A variável que compreende a percepção dos consumidores em relação a se o preço estabelecido está em conformidade com a qualidade dos produtos e serviços oferecidos (v18) apresenta maior peso no construto valor percebido.

A variável 4 é responsável pelo maior impacto no construto imagem, a qual corresponde à preocupação da empresa com os seus clientes. A questão sobre a satisfação dos 
clientes em relação aos serviços prestados pelo fast food preferido (v19) corresponde ao peso mais elevado no construto satisfação. Quanto ao construto lealdade, a variável referente à possibilidade do consumidor recomendar a sua empresa favorita de fast food a algum amigo (v30) foi responsável pelo maior peso.

A razão crítica calculada para todos os coeficientes é superior ao valor crítico tabelado (1,96), mostrando que todos os coeficientes são significativos, o que apoia a base teórica para designação dos indicadores em cada construto. Verifica-se que todos os coeficientes das variáveis observadas assumiram valores positivos, resultado coerente com o modelo teórico utilizado de Tenenhaus et al. (2005). As cargas fatoriais estimadas através da técnica de bootstrap apresentaram coeficientes bem próximos aos estimados para a amostra original indicando a consistência dos coeficientes.

Com o objetivo de validar globalmente a utilização do modelo PLS-PM nesse trabalho, procedeu-se a análise do Goodness of Fit - GoF (Tabela 5). A literatura recomenda que o índice de ajuste GoF seja superior a 0,9 para que o modelo seja considerado ajustado. Os índices de GoF para os modelos interno e externo, alcançaram o valor recomendado, mostrando que a aplicação do método PLS-PM foi adequado.

Tabela 5 - Goodness of Fit (GoF)

\begin{tabular}{l|c|c|c|c}
\hline & GoF & GoF (Bootstrap) & Erro padrão & Razão crítica (CR) \\
\hline Absoluto & 0,525 & 0,531 & 0,021 & 24,456 \\
\hline Relativo & 0,922 & 0,905 & 0,014 & 66,872 \\
\hline Modelo externo & 0,997 & 0,995 & 0,001 & 842,858 \\
\hline Modelo interno & 0,925 & 0,910 & 0,013 & 68,993 \\
\hline
\end{tabular}

Fonte: Elaborado pelos autores, 2012.

A avaliação da validade do modelo é apresentada na Tabela 6, assim como os dados para confirmação das quatro primeiras hipóteses do estudo. $O$ coeficiente de determinação $R^{2}$ foi de 0,663 , o que mostra que as variáveis em conjunto explicam $66,3 \%$ da satisfação, um valor, que conforme o critério enfatizado por Chin (1998) indica um coeficiente de determinação substancial. Os coeficientes obtidos com as variáveis latentes: imagem, qualidade e valor percebido foram significativos. A expectativa dos clientes não é significativa em função de apresentar razão crítica com valor menor que 1,96. 
Dessa forma, o construto que mais influencia na variação total da satisfação é o valor percebido, correspondendo a $43,4 \%$ do total explicado pelas variáveis enfatizadas, as quais consideram se o preço está em conformidade com a qualidade dos produtos e serviços e, a avaliação pelos clientes da empresa preferida em relação às demais organizações.

Tabela 6 - Path Coefficients e $\mathrm{R}^{2}$ da Satisfação

\begin{tabular}{l|c|c|c|r}
\hline \multicolumn{1}{c|}{ Variável latente } & Valor & Valor (Bootstrap) & $\begin{array}{c}\text { Erro padrão } \\
\text { (Bootstrap) }\end{array}$ & $\begin{array}{c}\text { Razão crítica } \\
\text { (CR) }\end{array}$ \\
\hline Intercepto & 0,000 & 0,000 & 0,000 & 0,054 \\
\hline Imagem & 0,153 & 0,157 & 0,053 & 2,904 \\
\hline Expectativa dos clientes & 0,062 & 0,067 & 0,062 & 1,004 \\
\hline Qualidade & 0,284 & 0,279 & 0,042 & 6,733 \\
\hline Valor percebido & 0,434 & 0,433 & 0,059 & 7,311 \\
\hline $\mathrm{R}^{2}$ & 0,663 & 0,673 & 0,037 & 17,831 \\
\hline
\end{tabular}

Fonte: Elaborado pelos autores, 2012.

Conforme o referencial teórico apresentado anteriormente, o valor percebido se refere à avaliação geral pelo consumidor da utilidade de um produto baseado em percepções do que é recebido e do que é dado, ou seja, o nível entendido de qualidade do produto em relação ao preço pago. Nesse sentido, as empresas provedoras dos serviços alimentícios considerados, podem potencializar seus resultados ao considerar que o cliente analisa a relação custo/benefício no momento de optar por seu lanche ou empresa.

A segunda dimensão que mais influencia na satisfação é a qualidade percebida, que contribui com $28,4 \%$ e, em terceiro lugar, a variável imagem participa com aproximadamente $15 \%$ no impacto da satisfação. Na sequência, a expectativa dos clientes aparece com apenas $6 \%$ de impacto na satisfação, no entanto, esta não é considerada como significante nesse estudo.

A partir dessas constatações é possível analisar a validade das hipóteses do estudo. A primeira hipótese afirmava que "a imagem influencia positivamente a satisfação dos clientes". Os resultados demonstraram que a relação é significativa e diretamente proporcional $\left(R^{2}=0,15\right)$, ou seja, quanto maior a imagem percebida da empresa, maior será a satisfação dos consumidores. Desta forma, confirmasse $\mathrm{H} 1$.

A Hipótese 2 afirmava que "a expectativa dos clientes influencia positivamente a satisfação dos clientes". Os dados mostram que a hipótese foi rejeitada, pois não foi considerada significativa para o estudo (razão crítica inferior a 1,96). Conforme a definição de Johnson et al. 
(2001), a expectativa do cliente é relacionada a sua experiência anterior com os produtos e serviços de uma empresa, além da percepção da propaganda. Dessa forma, é possível inferir que os consumidores pesquisados não levam em consideração a experiência anterior, mas sim a imagem, qualidade e valor percebido da empresa.

A terceira hipótese propunha que "qualidade percebida influencia positivamente a satisfação dos clientes". Os resultados demonstraram que há uma relação positiva de dependência entre os construtos $\left(R^{2}=0,28 ; p=0,000\right)$, ou seja, comprova-se a influência da qualidade na satisfação. A Hipótese 4 aborda que "o valor percebido influencia positivamente a satisfação dos clientes", e esta foi a relação mais forte estabelecida $\left(R^{2}=0,43\right)$, ou seja, quanto maior for o valor percebido pelo cliente, mas satisfeito ele se tornará.

A Tabela 7 apresenta os resultados para regressão do construto reclamações como dependente da satisfação dos clientes a fim de confirmar a Hipótese 5. A satisfação dos clientes mostrou-se significativa na explicação de $26,9 \%$ da variação das reclamações. Desse modo, é possível validar a Hipótese 5 em que a satisfação dos clientes influencia positivamente as reclamações, no sentido de que os clientes satisfeitos tendem a reclamar menos dos serviços de fast food.

Tabela 7 - Impacto e contribuição das variáveis a Reclamação

\begin{tabular}{|c|c|c|c|c|c|c|c|}
\hline $\begin{array}{l}\text { Variável } \\
\text { latente }\end{array}$ & $\begin{array}{c}\text { Coeficiente } \\
\text { Padronizado }\end{array}$ & $\begin{array}{c}\text { Coeficiente } \\
\text { Padronizado } \\
\text { Bootstrap }\end{array}$ & $\begin{array}{c}\text { Erro padrão } \\
\text { Bootstrap }\end{array}$ & $\begin{array}{l}\text { Razão } \\
\text { crítica }\end{array}$ & Correlação & $\begin{array}{l}\text { Correlação x } \\
\text { Coeficiente }\end{array}$ & $\begin{array}{c}\text { Contribuição } \\
\text { ao } R^{2}(\%)\end{array}$ \\
\hline Satisfação & 0,518 & 0,516 & 0,051 & 10,04 & 0,518 & 0,269 & 100 \\
\hline $\mathbf{R}^{2}$ & 0,269 & 0,268 & 0,052 & & & & \\
\hline
\end{tabular}

Fonte: Elaborado pelos autores, 2012.

A Tabela 8 relaciona o impacto e a contribuição das variáveis latentes, imagem e satisfação em relação à variável lealdade, no intuito de analisar as hipóteses 6 e 7. Constata-se que a imagem exerce um impacto positivo e significativo sobre a lealdade na ordem de 0,26 . De forma semelhante, a variável reclamações exibiu um vínculo positivo com a variável lealdade na ordem de 0,28 , o que corrobora a Hipótese 7 (as reclamações influenciam positivamente a lealdade dos clientes), ou seja, o número de reclamações influencia o grau de satisfação. A satisfação dos clientes exerce um impacto positivo de 0,343 sobre a lealdade, confirmando a Hipótese 6 (a satisfação dos clientes impacta positivamente a lealdade dos mesmos), o que significa que $34,3 \%$ 
da lealdade dos clientes pode ser explicada pela sua satisfação, corroborando a abordagem de vários autores que consideram a satisfação como predizente da lealdade.

Tabela 8 - Impacto e contribuição das variáveis a Lealdade

\begin{tabular}{|c|c|c|c|c|c|c|c|}
\hline $\begin{array}{l}\text { Variável } \\
\text { latente }\end{array}$ & $\begin{array}{l}\text { Coeficiente } \\
\text { Padronizado }\end{array}$ & $\begin{array}{c}\text { Coeficiente } \\
\text { Padronizado } \\
\text { Bootstrap }\end{array}$ & $\begin{array}{c}\text { Erro } \\
\text { padrão } \\
\text { Bootstrap } \\
\end{array}$ & $\begin{array}{l}\text { Razão } \\
\text { crítica }\end{array}$ & Correlação & $\begin{array}{c}\text { Correlação } \\
\text { x } \\
\text { Coeficiente }\end{array}$ & $\begin{array}{c}\text { Contribuição } \\
\text { ao } \mathbf{R}^{2}(\%)\end{array}$ \\
\hline Imagem & 0,260 & 0,259 & 0,020 & 12,68 & 0,519 & 0,135 & 25,414 \\
\hline Satisfação & 0,343 & 0,342 & 0,021 & 16,09 & 0,686 & 0,236 & 44,349 \\
\hline Reclamação & 0,284 & 0,289 & 0,018 & 15,22 & 0,566 & 0,161 & 30,235 \\
\hline $\mathbf{R}^{2}$ & 0,532 & 0,535 & 0,043 & & & & \\
\hline
\end{tabular}

Fonte: Elaborado pelos autores, 2012.

Complementando a análise a respeito da equação de regressão do construto lealdade, constata-se que as três variáveis exercem impacto semelhante na contribuição ao coeficiente de determinação $R^{2}$ da equação que explicou $53,2 \%$ da lealdade. Das sete hipóteses levantadas pelo estudo, seis foram confirmadas, conforme o Quadro 1.

Quadro 1 - Resultado das hipóteses do estudo

\begin{tabular}{|l|l|}
\hline Hipótese & Resultado \\
\hline H1 - A imagem influencia positivamente a satisfação dos clientes. & Confirmada \\
\hline H2 - A expectativa dos clientes influencia positivamente a satisfação. & Rejeitada \\
\hline H3 - A qualidade percebida influencia positivamente a satisfação dos clientes. & Confirmada \\
\hline H4 - O valor percebido influencia positivamente a satisfação dos clientes. & Confirmada \\
\hline H5 - A satisfação dos clientes influencia positivamente as reclamações. & Confirmada \\
\hline H6 - A satisfação dos clientes influencia positivamente a lealdade deles & Confirmada \\
\hline H7 - As reclamações influenciam positivamente a lealdade dos clientes. & Confirmada \\
\hline
\end{tabular}
Fonte: Elaborado pelos autores, 2012.

A satisfação é considerada por Anderson e Mittal (2000) como preditora do desempenho organizacional, contribuindo para a retenção de clientes e aumento dos lucros da empresa. Assim, a mensuração da satisfação dos clientes mostra-se uma ferramenta para alcance de melhores resultados das empresas, ao permitir avaliar quais os construtos são mais importantes para os consumidores, ao passo que as organizações, ao centrar seus esforços nessas dimensões podem atingir a satisfação e retenção de clientes. Através do estudo realizado, percebe-se que os jovens consumidores levam em consideração o valor percebido, a imagem e a qualidade como antecedentes da satisfação no momento de optar pela empresa de fast food, sendo que a satisfação impacta na lealdade e no índice de reclamações. Observa-se que a 
dimensão imagem, apesar de não apresentar validade convergente, possui validade discriminante, sendo considerada como determinante da satisfação.

\section{CONSIDERAÇÕES FINAIS}

O artigo teve como objetivo mensurar o nível de satisfação dos clientes com o serviço de fast food, compreendendo as variáveis expectativas dos clientes, qualidade percebida, imagem, valor percebido, reclamações e lealdade. A coleta de dados foi realizada por meio de questionários aplicados aos acadêmicos da Universidade Federal de Santa Maria e os dados foram analisados pelo método PLS-PM.

Os resultados encontrados no trabalho atenderam às hipóteses e ao objetivo propostos, demonstrando que a satisfação dos clientes de fast food é influenciada pela imagem, qualidade e valor percebidos. A satisfação impacta positivamente nas reclamações, considerando que se os consumidores estão satisfeitos tendem a reclamar menos dos serviços; e impacta positivamente na lealdade, pois o cliente tende a permanecer comprando os produtos da empresa que está satisfazendo seus desejos e suas necessidades. A expectativa dos clientes foi relacionada positivamente com a satisfação, mas não apresentou significância para o estudo e, por isso, a Hipótese 2 foi rejeitada.

Os resultados obtidos corroboram outros estudos realizados, como a pesquisa de Leite e Filho (2007), que resultou na satisfação como influenciada positivamente pelo valor percebido, pelas expectativas, pela qualidade e pela imagem. No entanto, a presente investigação proporcionou novas contribuições, uma vez que as expectativas dos clientes não apresentaram significância para o estudo e o construto valor percebido destacou-se como o principal responsável pela satisfação dos clientes em relação aos serviços de fast food. Para alguns autores, o valor percebido antecede a satisfação, possibilitando a avaliação da mesma e conforme Kotler (1998), a satisfação é função do desempenho percebido e das expectativas, ou seja, é necessário que o desempenho atenda às expectativas para que o consumidor fique satisfeito. Assim, o valor percebido compreende o resultado da empresa referente ao produto e/ou serviço oferecido ao cliente. 
Estudos sobre fast food mostram-se relevantes em função do referido segmento apresentar ascensão, nos últimos anos, decorrente das mudanças que modificam o hábito de alimentação das pessoas, com destaque ao aumento das refeições não realizadas em casa (STORCK et al., 2003). Dessa forma, restaurantes e lanchonetes são buscados pelos indivíduos no intuito de sanar a necessidade de sua alimentação e, visando a alcançar facilidade e rapidez, o fast food surge como alternativa.

A presente pesquisa propicia contribuições na área da gestão, no sentido que permite identificar quais variáveis mais influenciam a satisfação dos consumidores, especialmente de fast food, mostrando em qual direção devem ser centrados os esforços dos gestores das empresas do referido segmento de alimentos, a fim de satisfazer as necessidades e desejos do público-alvo. Conforme se demostrou no estudo, a satisfação é imprescindível para as empresas, pois é considerada preditora do desempenho organizacional, contribuindo para a retenção de clientes e aumento dos lucros da organização. Com o desenvolvimento dessa investigação, foi possível verificar quais construtos são mais importantes na determinação da satisfação dos consumidores (valor percebido, imagem e qualidade) e que devem ser potencializados pelas empresas, já que as mesmas podem maximizar seus lucros a partir da satisfação.

Ademais, o ambiente concorrencial em que as organizações atuam, em que a oferta de produtos e serviços, torna-se cada vez maior e com a facilidade de disponibilidade às pessoas, faz com que as empresas busquem novas formas de atender às necessidades dos clientes com qualidade e se diferenciar dos concorrentes. Esse processo perpassa pela análise da satisfação dos clientes, o que fortalece estudos da natureza como a presente pesquisa.

A partir das análises realizadas, verifica-se que existem outras variáveis além das presentes no modelo que devem ser investigadas. $O$ estudo realizado apresentou limitação referente à coleta de dados, a qual consistiu em amostra homogênea por compreender apenas acadêmicos da Universidade Federal de Santa Maria/RS. Outra limitação se refere à incipiência de trabalhos já realizados sobre fast food, sendo que na literatura são encontrados poucos estudos nesse campo. Diante disso, futuras pesquisas podem considerar uma maior amostra, de locais diferentes e com públicos variados, a fim de alcançar melhores resultados. Sugere-se, ainda, a investigação de outros segmentos que atendem o público-jovem, pois os jovens serão 
consumidores durante vários anos e seu estudo é de extrema valia para os gestores e pesquisadores, quanto às tendências de consumo.

\title{
MEASUREMENT OF SATISFACTION CUSTOMERS WITH FAST FOOD SERVICES
}

\begin{abstract}
Customer satisfaction has become one of the most relevant aspects for companies due to the increase consumer requirements who passed to enjoy a growing range of products and services. In this context, several studies were conducted to create and refine models, like the Swedish Customer Satisfaction Index, American Customer Satisfaction Index and European Customer Satisfaction Index, to identify the customer satisfaction level. This article aims to measure the customer satisfaction level with the fast food service, considering the customer expectations, perceived quality, image, perceived value, loyalty and complaints, which are part of the European Index of Consumer Satisfaction. The research held is descriptive and the data collection was obtained through a questionnaire consisting of 30 questions, applied students to 210 in Santa Maria, Rio Grande do Sul state, Brazil. Data analysis was made using the PLS-PM method. The results show that the perceived value is the variable what has most impact on customer satisfaction and then the perceived quality and image and customers' expectations. Most of the research hypotheses were confirmed, so than, the model used proved to be suitable for evaluating customers satisfaction regarding the fast food services.
\end{abstract}

Keywords: Fast-Food. Partial Least Squares. Customer Satisfaction.

\section{REFERÊNCIAS}

ANDERSON, E. W.; FORNELL, C. A customer satisfaction research prospectus. In: RUST, R. T.; OLIVER, R. L. (Eds.). Service quality: new directions in theory and practice. Thousand Oaks: Sage Publications, p. 241-68, 1994.

ANDERSON, E. W.; MITTAL, V. Strengthening the Satisfaction-Profit Chain. Journal of Service Research, v. 3, n. 2, p. 107-120, 2000. 
ANDREASSEN, T. W.; LINDESTAD, B. The effect of corporate image in the formation of customer loyalty. Journal of Service Marketing, v.1, p. 82-92, 1998.

BARICH, H.; KOTLER, P. A framework for marketing image management. Sloan Management Review, p. $94-104,1991$.

BOULDING, W. et al. A dynamic process model of service quality: from expectations to behavioral intentions. Journal of Marketing Research, v. 30, p.07-27, 1993.

CHIN, W. W. The partial least squares approach to structural equation modeling. In: Modern Methods for Business Research. Mahwah, NJ: Lawrence Erlbaum Associates, p. 295-358, 1998.

CHITTY, B.; SOUTAR, G.N. Is the ECSI model applicable to tertiary education? ANZMAC Conference, Wellington, New Zealand, 2004.

CORRAR, L. J. et al. Análise Multivariada: para os Cursos de Administração, Ciências Contábeis e Economia. FIPECAFI - Fundação Instituto de Pesquisa Contábeis, Atuárias e Financeiras. São Paulo: Atlas, 2006.

DESCHAMPS, J. P.; NAYAK, P. R. Produtos irresistíveis - como operacionalizar um fluxo perfeito de produtos do produtor ao consumidor. São Paulo: Makron Books, 1996.

DEVLIN, S. J.; DONG, H. K.; BROWN, M. Selecting a Scale for Measuring Quality, Marketing Research. A Magazine of Management \& Applications, v. 5, n. 1, p. 12-17, 1993.

FORNELL, C. A national customer satisfaction barometer: the Swedish experience. Journal of Marketing, v. 56, p. 6-21, 1992.

. National and corporate customer satisfaction indexes. A presentation at the World. Quality Day. Amsterdam: World Trade Center, 1991.

FORNELL, C et al. The American Customer Satisfaction Index: Nature, purpose and findings. Journal of Marketing, v. 60, p.7-18, 1996.

FORNELL, C.; LARCKER, D. F. Structural equation models with unobservable variables and measurement error: algebra and statistics. Journal of Marketing Research, v.18, n.3, p. 328-388, 1981.

GALE, B. T. Gerenciando o valor do cliente: criando qualidade e serviços que os clientes podem ver. São Paulo: Pioneira, 1996.

GRÖNROOS, C. Service management and marketing: managing the moment of truth in service competition. Lexington : Free Press, Lexington Books, 1990.

HAIR, J.R. et al. Fundamentos de métodos de pesquisa em administração. Porto Alegre: Bookman, 2005. 
HIRSCHMAN, A. O. Exit, voice, and loyalty - responses to decline in firms, organizations, and states. Boston, MA: Harvard University Press, 1970.

HUFF, L.; FORNELL, C.; ANDERSON, E. W. Quality and productivity: contradictory and complementary. Working Paper, NQRC (National Quality Research Center): The University of Michigan, 1994.

JOHNSON, M. D. et al. The evolution and future of national customer satisfaction index models. Journal os Economic Psychology, v. 22, p. 217-245, 2001.

JONES, T. O.; SASSER, W. E. Why satisfied customers defect. Harvard Business Review, p. 88-99, 1995.

KOTLER, P. Administração de marketing: análise, planejamento, implementação e controle. São Paulo: Atlas, 1998.

KRISTENSEN, K.; MARTENSEN, A.; GRONHOLDT, L. Customer satisfaction measurement at post denmark: results of application of the European customer satisfaction index methodology. Total Quality Management, v.11, n. 7, p. 1007-1015, 2000.

LEITE, R. S.; FILHO, C. G. Um estudo empírico da aplicação do índice europeu de satisfação do cliente (ECSI) no Brasil. Revista de Administração Mackenzie, v. 8, n. 4, p. 178-200, 2007.

LOPES, H. E. G.; PEREIRA, C. C. de P.; VIEIRA, A. F. S. Comparação entre os modelos norteamericano (ACSI) e europeu (ECSI) de satisfação do cliente: um estudo no setor de serviços. RAM Revista de Administração Mackenzie, v. 10, n. 1, p. 161-186, 2009.

LOUGHLIN, C.; COENDERS, G. Aplication of the european customer satisfaction index to postal services structural equation models versus partial least squares. Working paper, Department of Economics, University of Girona, 2002.

MALHOTRA, N. Pesquisa de marketing: orientação aplicada. Porto Alegre: Bookman, 2001.

MILAN. G, S.; TREZ. G. Pesquisa de satisfação: um modelo para planos de saúde. RAE eletrônica, São Paulo, v. 4 n. 2, 2005.

MOREIRA, D. A. Dimensões do desempenho em manufatura e serviços. São Paulo: Pioneira, 1996.

MOWEN, J. C.; MINOR, M. Consumer behavior. 5. ed. Upper Saddle River: Prentice-Hall, 1998.

NETO, L. C. B.; COSTA, J. A. F.; PESSOA, H. F. C. Gestão da satisfação e lealdade do cliente: um estudo dos fatores que influenciam na satisfação e lealdade dos clientes corporativos de telefonia celular. In: SIMPÓSIO DE ADMINISTRAÇÃO DA PRODUÇÃO, LOGÍSTICA E OPERAÇÕES INTERNACIONAIS, 12, 2009, São Paulo. Anais... São Paulo: FGV-EAESP, 2009. 
NGUYEN, N.; LEBLANC, G. Corporate image and corporate reputation in customers retention decisions in services. Journal of Retailing and Consumer Services, v.8, n.4, p. 227-236, 2001..

OLIVER, R. L. Satisfaction: a behavioral perspective on the consumer. New York: McGraw-Hill, 1997. Whence consumer loyalty? Journal of Marketing, v. 63, 1999.

PROENÇA, R. P. C. Inovação tecnológica na produção de alimentação coletiva. Santa Catarina: Insular, p. 15-50, 1997.

REICHHELD, F. F. The One Number You Need to Grow. Harvard Business Review, p. 46-54, 2003.

RICHINS, M. L. Negative Word-of-mouth by Dissatisfied: a Pilot Study. Journal of Marketing, v. 47, p. 68-78, 1983.

RODRIGUES, J. M. S. Gestão da satisfação e da fidelidade de consumidores: um estudo dos fatores que afetam a satisfação e a fidelidade no mercado de turismo. 2003. 72 f. Tese (Mestrado em Ciências em Engenharia da Produção) - Universidade Federal do Rio Grande do Norte, Natal, 2003.

SCHNEIDER, B.; BOWEN, D. E. Understanding customer delight and outrage. Sloan Management Review, p. 35-45, 1999.

SHETH, J.; MITTAL, B.; NEWMAN, B. Comportamento do cliente: indo além do comportamento do consumidor. São Paulo: Atlas, 2001.

STORCK, C. R. et al. Monitoramento da temperatura de preparações quentes e frias em restaurantes sel-service, na Zona Urbana de Santa Maria. Revista Nutrição em Pauta, São Paulo, ano XI, n. 59, 2003.

TENENHAUS, M. et al. PLS path modeling. Computational Statistics \& Data Analysis, v. 48, p. 159205, 2005.

TONI, D. de. et al. Análise da Satisfação com Instituições de Educação Superior e Imagem: Comparando Instrumentos. In: ENCONTRO NACIONAL DA ANPAD, 30, 2006, Salavador. Anais... Salvador: ANPAD, 2006.

URDAN, A. T.; RODRIGUES, A. R. O Modelo do índice de satisfação dos clientes norte- americano: um exame inicial no Brasil com equações estruturais. Revista de Administração Contemporânea, v. 3, n. 3, p. 109-130, 1999.

VEIGA, R. T. Um exame empírico do modelo de consequências comportamentais da qualidade de serviços. 2000. 420 f. Tese ( Doutorado em Administração de Empresas ) - Faculdade de Ciências Econômicas, Universidade Federal de Minas Gerais, Belo Horizonte, 2000. 
WEBSTER JUNIOR, F. E. Marketing driven management. New York: John Wiley \& Sons, 1994.

WITTINK, D. R.; BAYER, L. R. Statistical Analysis of Customer Satisfaction Data: Results from a National Experiment with Measurement Scales, Cornell University Johnson Graduate School of Management, 1994.

WOLD, H. O. Causal flows with latent variables: Partings of the ways in the light of NIPALS modeling. European Economic Review, v. 5, n. 1, p. 67-86, 1974.

WOLD, H. O. Soft modeling: the basic design and some extensions. In: K. G. Jöreskog \& H. O. Wold (eds), Systems under indirect observations, Part II (pp. 1-54). Amsterdan: North-Holland, 1982.

WOLD, H. O. Partial least squares. In: S. Kotz \& N. L. Johnson (Eds). Encyclopedia of statistical sciences, v. 6, p. 581-591, 1985.

ZEITHAML, V. Consumer Perceptions of Price, Quality, and Value: A means-end Model and Synthesis of Evidence, Journal of Marketing, Chicago, v. 52, n.3, p.2-22, 1988. 


\section{APÊNDICE A}

Variáveis Latentes com suas respectivas Variáveis Manifestas.

\begin{tabular}{|c|c|}
\hline VARIÁVEL LATENTE & VARIÁVEL MANIFESTA \\
\hline $\begin{array}{l}\text { Qualidade Percebida de } \\
\text { Produtos/Serviços }\end{array}$ & $\begin{array}{l}\text { Q11. Qualidade geral percebida? } \\
\text { Q12. Qualidade na prestação de serviços em geral? } \\
\text { Q13. Atendimento ao cliente e aos avisos pessoais oferecidos? } \\
\text { Q14. Qualidade dos serviços que você usa? } \\
\text { Q15. Variedade de produtos e serviços oferecidos? } \\
\text { Q16. Confiabilidade dos produtos e serviços prestados? } \\
\text { Q17. Clareza e transparência da informação fornecida sobre procedimentos gerais? }\end{array}$ \\
\hline Valor percebido & $\begin{array}{l}\text { Q. 18. Você acha que o preço estabelecido está conforme com a qualidade dos } \\
\text { produtos e serviços oferecidos pela empresa escolhida? } \\
\text { Q20. Levando em conta os serviços e produtos oferecidos por outras empresas, } \\
\text { como você avalia os serviços de fast food da empresa escolhida? }\end{array}$ \\
\hline Imagem & $\begin{array}{l}\text { Q1. A empresa é confiável naquilo que promete? } \\
\text { Q2. A empresa é estável e estabelecida.? } \\
\text { Q3. Você percebe que a empresa contribui com a sociedade? } \\
\text { Q4. Ela está preocupada constantemente com os clientes? } \\
\text { Q5. É inovadora e olha para o futuro? } \\
\text { Q6. Possui uma marca conhecida? } \\
\text { Q22. A empresa de fast food utilizam tecnologias e procedimentos modernos? } \\
\text { Q23. As instalações do fast food são modernas e visualmente atraentes? }\end{array}$ \\
\hline Expectativa dos clientes & $\begin{array}{l}\text { Q7. A qualidade esperada no momento em que você optou pelo fast food? } \\
\text { Q8. Sua expectativa que a empresa de fast food venha oferecer produtos e serviços } \\
\text { que atendam suas necessidades? } \\
\text { Q9. Você esperava ou espera ter problemas com of fast food? } \\
\text { Q10. Você esperava ou espera que o fast food cobre preços justos pelos serviços? }\end{array}$ \\
\hline Satisfação & $\begin{array}{l}\text { Q.19. Considerando as suas expectativas, você ficou satisfeito em relação aos } \\
\text { serviços prestados pelo fast food? } \\
\text { Q21. Considerando as suas expectativas, você ficou decepcionado em relação aos } \\
\text { serviços prestados pelo fast food? } \\
\text { Q24. Qual a sua avaliação quanto à satisfação geral com o fast food? } \\
\text { Q25. Relativo ao atendimento das suas expectativas qual sua avaliação? } \\
\text { Q26. Considerando uma empresa perfeita de fast food, como você avalia a empresa } \\
\text { escolhida? }\end{array}$ \\
\hline Lealdade & $\begin{array}{l}\text { Q28. Se você tivesse a possibilidade de escolher outra empresa, qual a } \\
\text { possibilidade de escolher novamente a que utiliza? } \\
\text { Q29. Supondo outra empresa de fast food competitiva que ofereça a mesma gama e } \\
\text { a qualidade dos produtos que sua empresa escolhida. Qual o percentual, em sua } \\
\text { opinião, que a outra empresa teria que reduzir seus preços para que você trocasse de } \\
\text { empresa? } \\
\text { Q30. Qual a possibilidade de você recomendar a sua empresa preferida de fast food } \\
\text { para um amigo? }\end{array}$ \\
\hline Reclamações & $\begin{array}{l}\text { Q27. Se você tivesse que reclamar com a empresa, como você acha que ele se } \\
\text { preocuparia com sua reclamação? }\end{array}$ \\
\hline
\end{tabular}

